\title{
hNUDT16: a universal decapping enzyme for small nucleolar RNA and cytoplasmic mRNA
}

\author{
Guangwen $\mathrm{Lu}^{1,2^{*}}$, Jie $\mathrm{Zhang}^{1^{*}}$, Yan $\mathrm{Li}^{1,2^{*}}$, Zhixin $\mathrm{Li}^{1,2}$, Na Zhang ${ }^{1,2}$, Xiang $\mathrm{Xu}^{3}$, Tingting Wang ${ }^{1}$, \\ Zhenhong Guan ${ }^{1}$, George F. Gao ${ }^{1,2,4}$, Jinghua Yan ${ }^{1 凶}$ \\ ${ }^{1}$ CAS Key Laboratory of Pathogenic Microbiology and Immunology (CASPMI), Institute of Microbiology, Chinese Academy of \\ Sciences (CAS), Beijing 100101, China \\ ${ }^{2}$ Graduate University, Chinese Academy of Sciences, Beijing 100049, China \\ ${ }^{3}$ College of Life Science, Anhui Agricultural University, Anhui 230036, China \\ ${ }^{4}$ Beijing Institutes of Life Science, Chinese Academy of Sciences, Beijing 100101, China \\ \ Correspondence: yanjh@im.ac.cn
}

Received January 10, 2011 Accepted January 19, 2011

\section{ABSTRACT}

Human NUDT16 (hNUDT16) is a decapping enzyme initially identified as the human homolog to the Xenopus laevis X29. As a metalloenzyme, hNUDT16 relies on divalent cations for its cap-hydrolysis activity to remove $\mathrm{m}^{7}$ GDP and $\mathrm{m}^{227}$ GDP from RNAs. Metal also determines substrate specificity of the enzyme. So far, only U8 small nucleolar RNA (snoRNA) has been identified as the substrate of hNUDT16 in the presence of $\mathrm{Mg}^{2+}$. Here we demonstrate that besides U8, hNUDT16 can also actively cleave the $m^{7}$ GDP cap from mRNAs in the presence of $\mathrm{Mg}^{2+}$ or $\mathrm{Mn}^{2+}$. We further show that hNUDT16 does not preferentially recognize U8 or mRNA substrates by our cross-inhibition and quantitative decapping assays. In addition, our mutagenesis analysis identifies several key residues involved in hydrolysis and confirms the key role of the REXXEE motif in catalysis. Finally an investigation into the subcellular localization of hNUDT16 revealed its abundance in both cytoplasm and nucleus. These findings extend the substrate spectrum of hNUDT16 beyond snoRNAs to also include mRNA, demonstrating the pleiotropic decapping activity of hNUDT16.

KEYWORDS hNUDT16, mRNA, U8 small nucleolar RNA, decapping activity, substrate specificity, subcellular localization

\section{INTRODUCTION}

Cells harbor an elaborately modulated system in order to strictly control the metabolism of cellular RNAs. Various nuclear and cytoplasmic events, such as DNA transcription, RNA capping, RNA splicing/processing, are actively involved in the process. Besides these systems a surveillance mechanism to identify and eliminate aberrant RNAs is also an intrinsic part of this elaborate machinery in maintenance and alteration of the steady-state balance of cellular RNAs (Dostie et al., 2000; Wilusz and Wilusz, 2004; Cougot et al., 2004b; Fenger-Grøn et al., 2005; Garneau et al., 2007; Sharma and Black, 2006). So far, two fundamental RNA decay pathways are known to exist, with the degradation occurring from either the 5 '-end or from the 3 '-end of the RNAs (Vasudevan and Peltz, 2003; Coller and Parker, 2004; Saguez et al., 2005). The $3^{\prime}-5^{\prime}$ mRNA decay occurs by deadenylation and the following complete degradation of the RNA. The latter event relies on the activity of the exosome, a multi-subunit complex with $3^{\prime}-5$ ' exonuclease activity (Anderson and Parker, 1998; Chen et al., 2001; Wang and Kiledjian, 2001; Mukherjee et al., 2002; Wang et al., 2002). While the $5^{\prime}-$ $3^{\prime}$ mRNA turnover pathway requires the removal of the $5^{\prime}$ $\mathrm{m}^{7}$ GpppG cap (Decker and Parker, 1994; Coller and Parker, 2004; Gu et al., 2004; Meyer et al., 2004; Parker and Song, 2004). This cap hydrolysis is carried out by the nucleoside diphosphatase hydrolases which can release $\mathrm{m}^{7} \mathrm{GDP}$ and leave a $5^{\prime}$-monophosphate at the $5^{\prime}$-end of the RNA. In cytoplasm, cap-removal could be performed by Dcp2 (Dunckley and Parker, 1999; van Dijk et al., 2002; Piccirillo

"These authors contributed equally to this work. 
et al., 2003). While in nucleus, NUDT16/X29 protein is reportedly capable of cleaving the 5 '-cap from intact RNAs (Tomasevic and Peculis, 1999; Ghosh et al., 2004; Peculis et al., 2007; Taylor and Peculis, 2008).

Dcp2 was initially identified in yeast as an RNA decapping protein (Dunckley and Parker, 1999). In vitro, Dcp2 was found to cleave all capped RNAs of more than 40 nucleotides in length. Metals such as $\mathrm{Mg}^{2+}$ and $\mathrm{Mn}^{2+}$ are required for hydrolysis (Dunckley and Parker, 1999, 2001; Tucker and Parker, 2000). In vivo, Dcp2 is concentrated in cytoplasmic foci, called processing bodies (P-bodies), where it functions in coordination with a variety of known or putative RNA decay factors(van Dijk et al., 2002; Cougot et al., 2004a). These findings demonstrate Dcp2 as a key factor involved in cytoplasmic RNA turnover. The identification of its homologous enzymes in mammals indicates the importance and universality of this RNA decay machinery in the cytoplasm (Lykke-Andersen, 2002; van Dijk et al., 2002; Wang et al., 2002; Piccirillo et al., 2003).

In the nucleus, RNA degradation also occurs. Degradation of mRNA in the nucleus (DNR) was identified as an mRNA decay system with both $3^{\prime}-5^{\prime}$ and $5^{\prime}-3^{\prime}$ decay activity, involving proteins such as exosome, Rrp6p, nuclear cap binding protein $\mathrm{Cbc} 1 \mathrm{p}$, and Xrn2/Rat1p (Das et al., 2000; Das et al., 2003; Kuai et al., 2005; Das et al., 2006). NUDT16/X29 has been identified as a mainly nucleus-localized enzyme with metal-dependent RNA decapping activity. It has a high affinity and specificity for binding and decapping U8 snoRNA in the presence of $\mathrm{Mg}^{2+}$. Moreover, the NUDT16/X29 protein is distinct from $D c p 2$, as Dcp2 can decap $m^{7} G$ capped RNAs in $\mathrm{Mn}^{2+}$ or $\mathrm{Mg}^{2+}$, but NUDT16/X29 only removes $\mathrm{m}^{7} \mathrm{G}$ and $\mathrm{m}^{227} \mathrm{G}$ caps present on many of small nuclear-limited RNAs in $\mathrm{Mn}^{2+}$ or $\mathrm{Co}^{2+}$ (Tomasevic and Peculis, 1999; Ghosh et al., 2004; Peculis et al., 2007).

Despite the difference in substrate specificity observed between Dcp2 and NUDT16/X29, there remains the intriguing possibility that the latter is part of the nuclear decapping machinery which parallels the cytoplasmic cap-removal system. Both enzymes belong to a large evolutionarily conserved family, Nudix hydrolases, which function as nucleotide diphosphatases acting upon various substrates $(\underline{X})(B h a t n a g a r$ et al., 1991; Bessman et al., 1996; Mildvan et al., 2005). Members of Nudix hydrolases are widespread among bacteria, virus, eukaryotes and archaea and are characterized by a highly conserved 23-amino acid Nudix motif, GX5EX7REUXEEXGU, where $U$ is an aliphatic or hydrophobic residue (Bessman et al., 1996). The motif is involved in catalysis and the coordination of $\mathrm{Mg}^{2+}, \mathrm{Mn}^{2+}$ or other divalent cations (Mildvan et al., 2005). NpnN, (d)NTP and capped RNAs are their substrates (Fisher et al., 2004; Hori et al., 2005; Ito et al., 2005).

hNUDT16 is the human homolog of NUDT16/X29 protein (Tomasevic and Peculis, 1999; Ghosh et al., 2004). Like Xenopus X29, different divalent metal ions determine the substrate specificity as well as the catalytic efficiency of this human decapping enzyme (Peculis et al., 2007). In $\mathrm{Mn}^{2+}$, the $\mathrm{m}^{7}$ GDP caps from both U8 and U3 snoRNAs could be efficiently removed by hNUDT16; whereas so far, only that of U8 was observed to be hydrolyzed in the presence of $\mathrm{Mg}^{2+}$ (Peculis et al., 2007). Results from Peculis showed that U8 snoRNA is involved in the processing of $5.8 \mathrm{~S}$ and $28 \mathrm{~S}$ rRNAs (Peculis and Steitz, 1993). Therefore, the ability of hNUDT16 to decap U8 in the presence of either metals indicates a role of this protein in the maturation of the large ribosome subunit through regulating stability of U8 snoRNA. In support of this, a recent report demonstrated that the enzyme is present in the nucleus (lyama et al., 2010).

Here we report that besides U8, hNUDT16 could also actively cleave the $m^{7}$ GDP cap from mRNA (such as luciferase mRNA and the mRNA encoding influenza NP protein) with either $\mathrm{Mg}^{2+}$ or $\mathrm{Mn}^{2+}$ being present. We further show that uncapped luciferase mRNA could efficiently compete against the hydrolysis of both substrates by the enzyme, indicating that hNUDT16 recognizes U8 and luciferase mRNA without preference. This viewpoint is further supported by a quantitative decapping assay which shows that hNUDT16 exhibits similar catalytic efficiencies toward different substrates. In addition, the mechanism of hydrolysis was investigated by mutation of residues in the consensus REXXEE motif. Furthermore, we demonstrated that hNUDT16 is ubiquitously present both in the cytoplasm and the nucleus. Taken together, these findings indicate hNUDT16 as a universal decapping enzyme for both small nucleolar RNAs and cytoplasmic mRNAs.

\section{RESULTS}

\section{The C-terminally His-tagged hNUDT16 is active in vitro}

The functional hydrolyzation of $\mathrm{m}^{7} \mathrm{GDP}$ from the 5 '-end of U8 snoRNA by hNUDT16 (originally referred to as H29K) has been reported previously (Ghosh et al., 2004). In this study, the enzyme subjected to the functional analysis was expressed as a C-terminally His-tagged protein as described in our last report (Zhang et al., 2008). To verify the catalytic activity of this recombinant enzyme, a TLC based decapping assay using U8 snoRNA as the substrate was designed (Ghosh et al., 2004). As expected, after the incubation of U8 snoRNA with the enzyme, an extra spot corresponding to $\mathrm{m}^{7} \mathrm{GDP}$ was observed in the migration profile, while the bovine serum albumin (BSA) control gave no such a band (Fig. 1A).

\section{hNUDT16 can remove $\mathrm{m}^{7}$ GDP from mRNA in vitro}

It has been reported that the decapping enzymes could display relaxed substrate specificity in the presence of specific metals. Apart from the canonical substrate-U8 
A

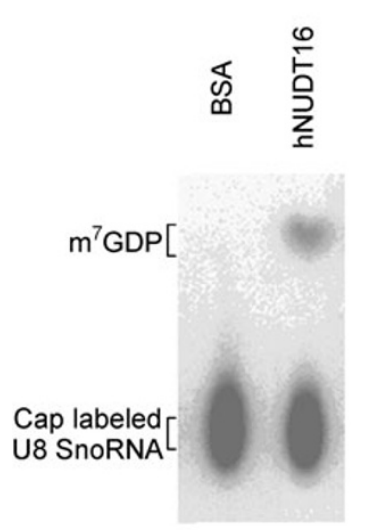

B

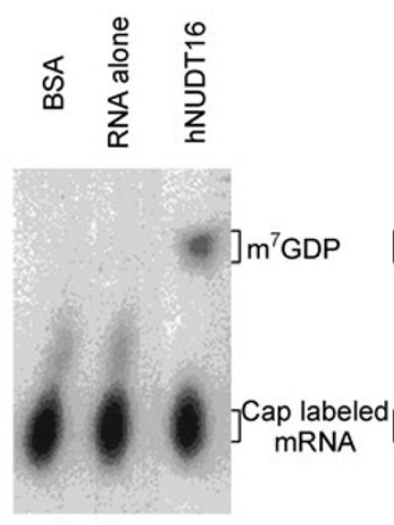

Luciferase
C

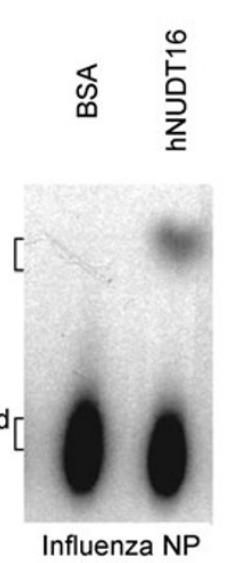

D

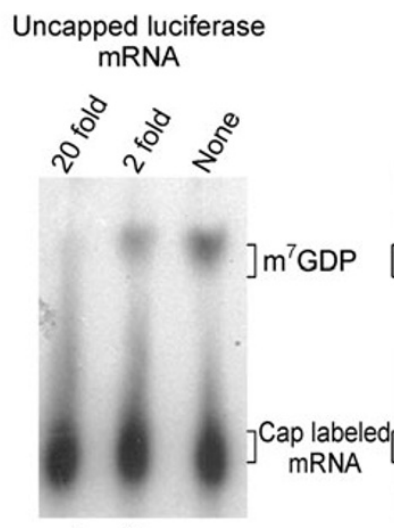

Luciferase
E

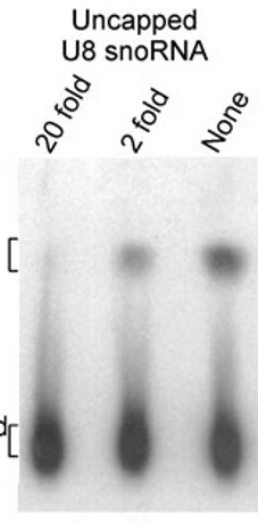

Luciferase

Figure 1. hNUDT16 could remove the cap structure from the 5'-end of mRNA in vitro. (A) The recombinant hNUDT16 was active in vitro. The decapping reactions were performed using $\mathrm{m}^{7} \mathrm{G}$-capped U8 snoRNA with irrelevant protein (BSA, bovine serum albumin) or purified hNUDT16 with $\mathrm{Mg}^{2+}$. (B and C) The cap-labeled luciferase mRNA or the mRNA encoding influenza NP protein, as indicated, was incubated with BSA, without or with hNUDT16 in the presence of $\mathrm{Mg}^{2+}$. The migration of the known standards is indicated. ( $D$ and $E$ ) Autoradiograph of the TLCs showing the competitive inhibition of the cap hydrolysis of luciferase mRNA by our enzyme using either uncapped luciferase mRNA (D) or uncapped U8 snoRNA (E). The competitors are added to the reaction at 20fold or 2-fold molar excess over the cap-labeled mRNA substrate.

snoRNA, other RNAs such as U3 snoRNA were also found to be decapped by hNUDT16 in $\mathrm{Mn}^{2+}$ (Peculis et al., 2007). Nevertheless $\mathrm{Mg}^{2+}$ did not endow the enzyme with the capability of cap removal from U3 snoRNA (Peculis et al., 2007). To address the question of whether hNUDT16 could catalyze cap hydrolysis of mRNAs, a luciferase mRNA as well as the mRNA coding for influenza NP protein was synthesized and cap labeled as described before (Tomasevic and Peculis, 1999). As shown in Fig. 1, after incubation of either of the two mRNA products with hNUDT16 in $\mathrm{Mg}^{2+}$, an unambiguous $\mathrm{m}^{7} \mathrm{GDP}$ band was observed. Neither BSA control nor the mRNA itself yielded such an $\mathrm{m}^{7} \mathrm{GDP}$ product (Fig. 1B and 1C). The amount of the cleaved product was comparable to that for U8 snoRNA (Fig. 1A), demonstrating an efficient decapping reaction for mRNAs occurred.

\section{The mRNA decapping activity of hNUDT16 could be competitively inhibited by uncapped mRNA and cross-inhibited by non-capped U8}

To further confirm the mRNA decapping capability of hNUDT16, a competitive inhibition assay was designed. The assay followed the standard TLC assay protocol except that the non-capped RNAs were mixed in advance with caplabeled luciferase mRNA before the addition of the enzyme (for details, see MATERIALS AND METHODS). Two different non-capped/capped RNA ratios (2:1 and 20:1) were selected. As shown in Fig. 1, the presence of 2-fold molar excess of non-capped luciferase mRNA over the cap-labeled substrate dramatically decreased the yield of $\mathrm{m}^{7} \mathrm{GDP}$ decapping product. At a non-capped/capped RNA ratio of $20: 1$, no obviously cleaved product could be detected within the time set (Fig. 1D). This result clearly showed that the non-capped luciferase mRNA was an effective competitor to the caplabeled mRNA substrate, and in turn demonstrated that the catalysis required the binding of the RNA moiety to the enzyme.

Since both U8 and mRNA were the substrates of hNUDT16 in vitro (see RESULTS above), to investigate whether noncapped U8 could cross-inhibit the decapping of mRNA and/or vice versa, we used uncapped U8 as a substitute for noncapped luciferase mRNA in the inhibition assay. As shown in Fig. 1E, when U8 was present, the decapping of luciferase mRNA was greatly inhibited. The overall inhibition pattern was similar to that observed for the non-capped luciferase mRNA. This phenomenon strongly indicated that hNUDT16 recognizes $\mathrm{U} 8$ and $\mathrm{mRNA}$ without preference, and therefore pointed to the same or at least partially overlapped spatial binding components for the RNA moieties of both substrates.

\section{Metal determines the efficiency of cap-hydrolysis catalyzed by hNUDT16}

Like other members of the Nudix family, hNUDT16 is a metalloenzyme whose activity is dependent on the presence of divalent cations (Ghosh et al., 2004; Peculis et al., 2007). Since all the decapping reactions above were performed under standard condition in the presence of $5 \mathrm{mM} \mathrm{Mg}^{2+}$, and in order to determine the effects of other metals on the enzymatic activity of the protein, a modified standard decapping assay was set up where magnesium was eliminated or replaced by different metals. As shown in 
Fig. 2, hNUDT16 without the coordination of metals could not catalyze the hydrolytic reaction since no detectable cleaved products could be observed for either the U8 snoRNA or the mRNA substrate. Both $\mathrm{Mg}^{2+}$ and $\mathrm{Mn}^{2+}$ could effectively switch the protein from apoenzyme to holoenzyme and $\mathrm{Mn}^{2+}$ was proved to be more efficient as the activating factor (Fig. 2). It is also noteworthy that apart from the hydrolyzed $\mathrm{m}^{7} \mathrm{GDP}$, a product of substantial amount whose migration pattern accords with that of GDP was observed in the presence of $\mathrm{Mn}^{2+}$ (Ghosh et al., 2004). This is probably due to the incorporation of RNAs with caps lacking 7-methyl group during our preparation of cap-labeled RNA substrates (see MATERIALS AND METHODS). These RNAs could only be decapped in much more active reactions when manganese was present. The other two divalent cations investigated were $\mathrm{Co}^{2+}$ and $\mathrm{Zn}^{2+}$. Both metals only confer limited hydrolytic capability on hNUDT16. After 30 min incubation of each of the three RNA substrates with the enzyme, only tiny amounts of $\mathrm{m}^{7} \mathrm{GDP}$ product were observed on the migration profiles (Fig. 2). In addition, Cobalt and Zinc act similarly in the enhancement of cap hydrolysis catalyzed by hNUDT16 and their effects were dramatically weaker than that of magnesium.

\section{hNUDT16 exhibits similar decapping efficiencies for U8 snoRNA and mRNA}

In our modified decapping assay, it is noteworthy that the cleaved cap products for either U8 or the two mRNAs were of comparable amounts for each of the four metals investigated, indicating similar decapping efficiencies of our enzyme toward these RNA substrates. Since all the assays performed above did not accurately control the amounts of input RNAs, we set up a new experiment to quantitatively compare the catalytic efficiency of our enzyme for different substrates. In this quantitative decapping assay, equal molars of either U8 snoRNA or luciferase mRNA were subjected to cap-

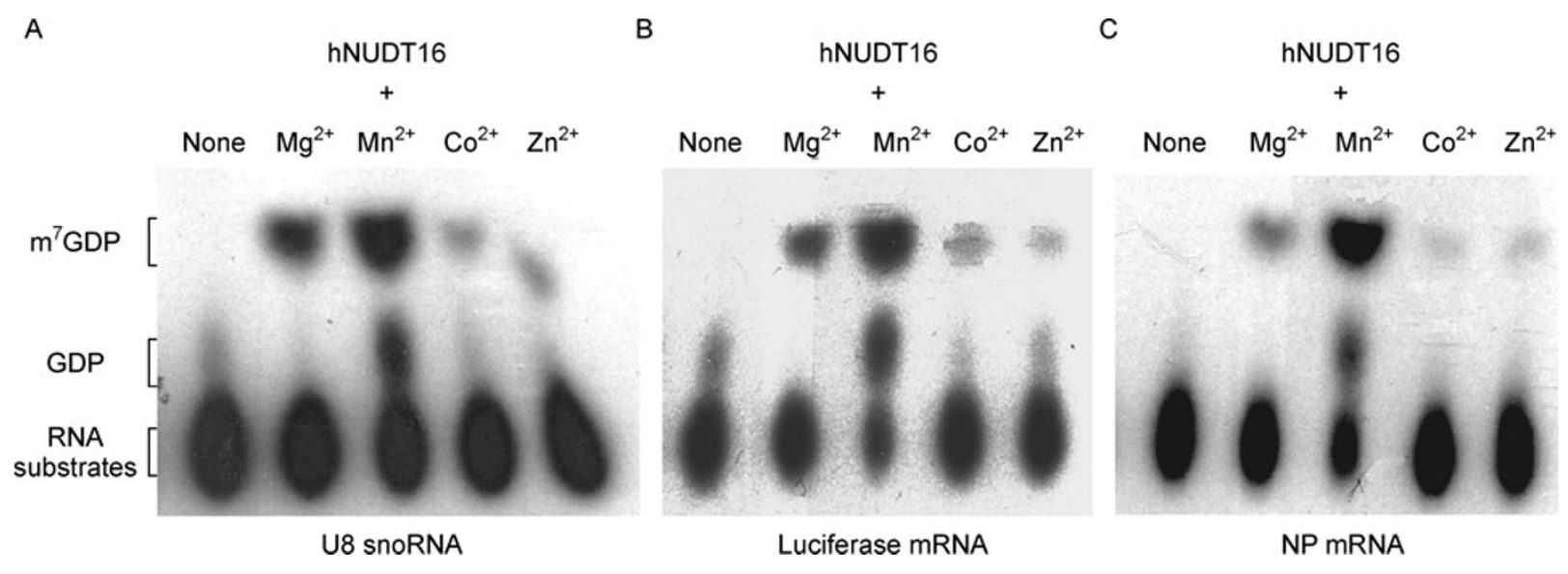

hydrolysis by hNUDT16 in the presence of $\mathrm{Mg}^{2+}$ (for details see MATERIALS AND METHODS). As shown in Fig. 3A, at each time point tested, $\mathrm{m}^{7} \mathrm{GDP}$ spots of similar size and intensity were observed for both substrates. Further quantitative calculation of the densities of these $m^{7} G D P$ spots confirmed the hydrolytic generation of similar amounts of the cap products from either RNAs (Fig. 3B). Therefore, this result demonstrated that hNUDT16 exhibits almost similar decapping efficiencies toward U8 and mRNA substrates.

\section{Mutagenesis analysis identifies several key residues involved in catalysis}

In Xeno X29, which shares over $50 \%$ sequence identity with hNUDT16, several key amino acids involved in the decapping reaction have been identified both by structural characterization and site directed mutagenesis analysis (Scarsdale et al., 2006; Peculis et al., 2007). These residues are mainly located within the Nudix box characterized by the sequence motif of GX5EX7REUXEEXGU (Bessman et al., 1996). Although the Nudix sequence motif in hNUDT16 is not in full accordance with the suggested pattern, we believe that it extends from Gly59 to Glu83. To define the possible amino acids that might be involved in the hydrolysis catalyzed by hNUDT16, four residues within the signature motif were selected and their mutant products (designated as R75L, E76Q, E79Q and E80Q, respectively) were overexpressed in Escherichia coli (E. coli). The purification procedure followed that for the wildtype protein, and the final purity of any of the four mutants was confirmed to be over $98 \%$ on sodium dodecyl sulfate polyacrylamide gel electrophoresis (SDS-PAGE) (Fig. 4B). The resultant enzymes were subjected to standard U8 snoRNA based TLC assay in paralleled experiments. Three residues were identified to be crucial in catalysis. As shown in Fig. 4A, the mutation of either Glu76 or Glu80 to Gln could almost totally abolish the enzyme's decapping activity under the standard assaying condition. The R75L mutant displayed

Figure 2. Metal determines the efficiency of cap-hydrolysis catalyzed by hNUDT16. Four metals including $\mathrm{Mg}^{2+}, \mathrm{Mn}^{2+}, \mathrm{Co}^{2+}$ and $\mathrm{Zn}^{2+}$ were investigated for their effects on the activation of hNUDT16. The decapping capability of our enzyme in the presence of different metals is shown for all the three RNA substrates. (A) U8 snoRNA; (B) luciferase mRNA; (C) influenza NP mRNA. 
A

U8 snoRNA

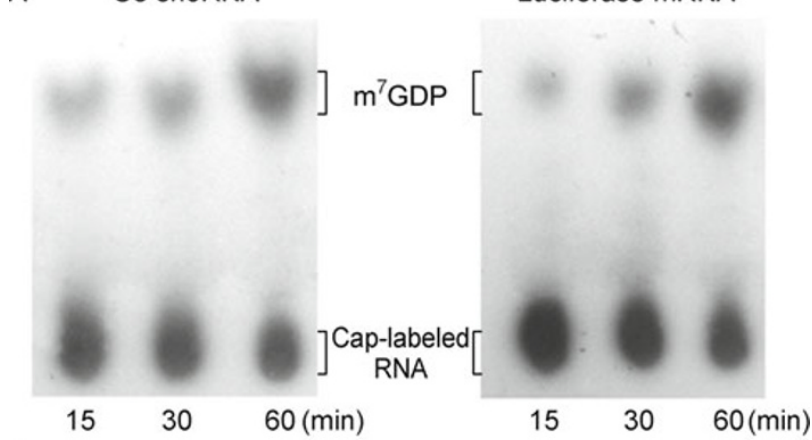

B

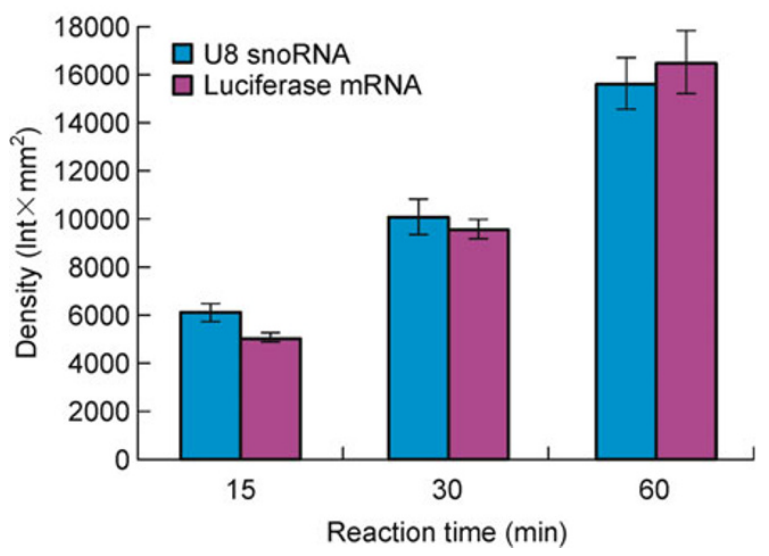

Figure 3. hNUDT16 exhibits similar decapping efficiencies toward different RNA substrates. (A) $0.2 \mathrm{nmol}$ of either cap-labeled U8 or luciferase mRNA was subjected to decapping reaction with magnesium being present. For both substrates, samples were pooled at different time points (15, 30 and $60 \mathrm{~min}$ ) and analyzed by TLC-based assay. A representative developing profile is shown. (B) The density of each $\mathrm{m}^{7} \mathrm{GDP}$ spot was calculated using Quantity One to represent the relative amount of the decapping product. This quantitative calculation confirms that at each time point, hNUDT16 could decap similar amounts of $\mathrm{m}^{7} \mathrm{GDP}$ from either U8 or luciferase mRNA substrate. The results were the average of three independent repetitions.

a dramatically weaker capability of hydrolysis, since only tiny amounts of cleaved product were observed on the migration profile. Another residue Glu79, the substitution of which by GIn also obviously decreased the decapping efficiency of the enzyme, should also play an important role in catalysis (Fig. 4A).

\section{hNUDT16 is of abundant presence in both cytoplasm and nucleus}

A recent report has demonstrated the presence of hNUDT16 in the nucleus (lyama et al., 2010). The identification of mRNA decapping activity for the enzyme urged us to also localize the protein in cells. We achieved this by expressing hNUDT16 with a myc-epitope at the C-terminus in HeLa cells and by detecting the tagged protein via immunofluorescence using an anti-myc antibody. As shown in Fig. 5A, the abundant presence of the enzyme was observed in both cytoplasm and nucleus. To exclude the possibility that the observed ubiquitous distribution of hNUDT16 in cells was the consequence of overexpression of the enzyme and/or the disturbance from the extra myc-tag, a specific polyclonal antibody was generated to detect the endogenous hNUDT16 and a similar subcellular distribution pattern for the enzyme was observed (Fig. 5B). We then quantitatively determined the relative amounts of hNUDT16 in the cytoplasm and the nucleus. The cyto- and nucle-fractionations of human embryonic kidney 293T cells were prepared, and equal amounts of the total input proteins for both were loaded on SDS-PAGE for Western-blot analysis using the hNUDT16 anti-sera. As shown in Fig. 5C, comparable amounts of this human decapping enzyme were detected in both the cytoplasm and the nucleus fractions. These results demonstrate that unlike its Xenopus counterpart which is mainly nucleolus-localized (Ghosh et al., 2004), hNUDT16 is almost ubiquitously present throughout the cell.

Surprisingly, we found that the concentration of Triton X100 , which we used to permeabilize cells, could dramatically influence the immunofluorescent results. After expression of the myc-tagged hNUDT16 in HeLa cells, treatment of the cells with $0.1 \%$ Triton $\mathrm{X}-100$ for 5 min (for details see MATERIALS AND METHODS) revealed predominantly cytoplasmic localization of hNUDT16 protein (Fig. 5D). However, with a higher concentration of Triton X-100 (0.5\%), abundant hNUDT16 enzyme was also detected in the nucleus (Fig. 5A).

\section{DISCUSSION}

hNUDT16 was first reported by Ghosh et al. as the human homologous counterpart protein to X29, the prototypical U8 small nucleolar RNA decapping hydrolase initially identified in Xenopus (Tomasevic and Peculis, 1999; Ghosh et al., 2004). Here we further characterized the in vitro catalytic activity of this human decapping enzyme. The protein's capabilities of cap removal from mRNAs in the presence of either $\mathrm{Mg}^{2+}$ or $\mathrm{Mn}^{2+}$ are reported in this study. We also demonstrated the enzyme is abundant in both cytoplasm and nucleus. Collectively, these findings extend the substrate spectrum of hNUDT16 and shed further light on the functional characteristics of this enzyme.

\section{Arginine within the REXXEE motif is also essential for efficient hydrolysis catalyzed by members of NUDT16 protein subfamily}

In X29, the key roles that the glutamic acids of the REXXEE motif play in catalysis have been elucidated by mutagenesis analysis. Peculis et al. showed that mutation of either E89 or E93 of the motif could abolish the activity of the enzyme, while 
A

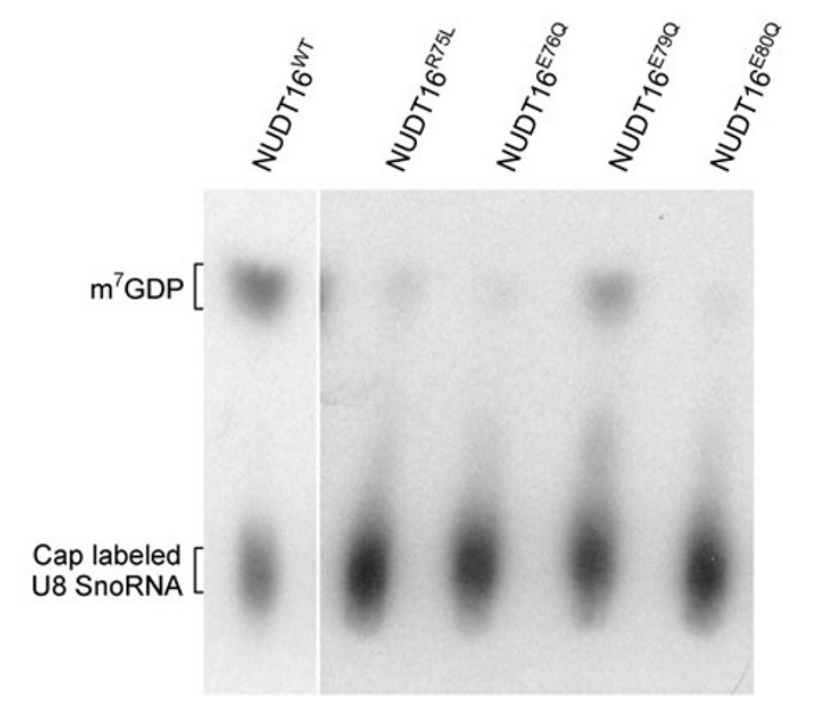

B

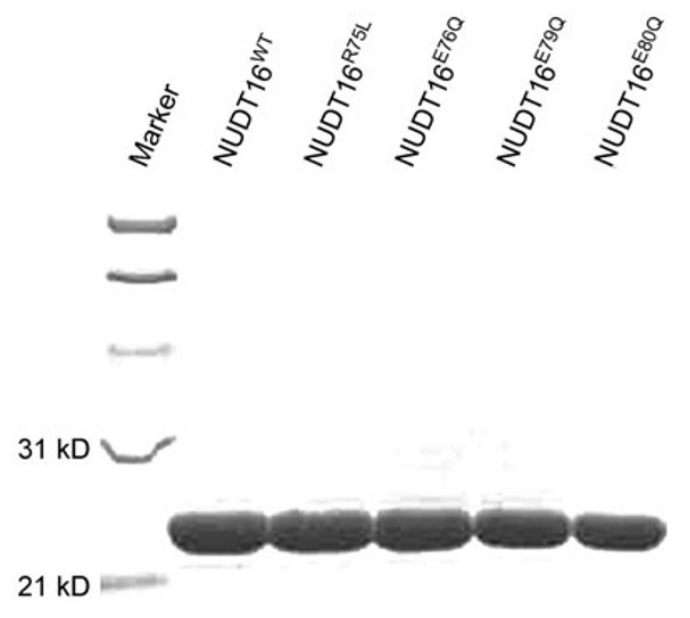

Figure 4. Mutagenesis analysis identified several key residues involved in cap-hydrolysis. (A) $\mathrm{m}^{7} \mathrm{G}$ cap-labeled $\mathrm{U} 8$ was incubated with either the WT protein or one of the four mutants in the presence of $\mathrm{Mg}^{2+}$ at $37^{\circ} \mathrm{C}$ for $30 \mathrm{~min}$. The result of the TLC development is shown. (B) Migration profiles of purified wild-type hNUDT16 and its four mutant proteins on sodium dodecyl sulfate polyacrylamide gel electrophoresis (SDS-PAGE). The standard molecular weight markers are indicated.

A

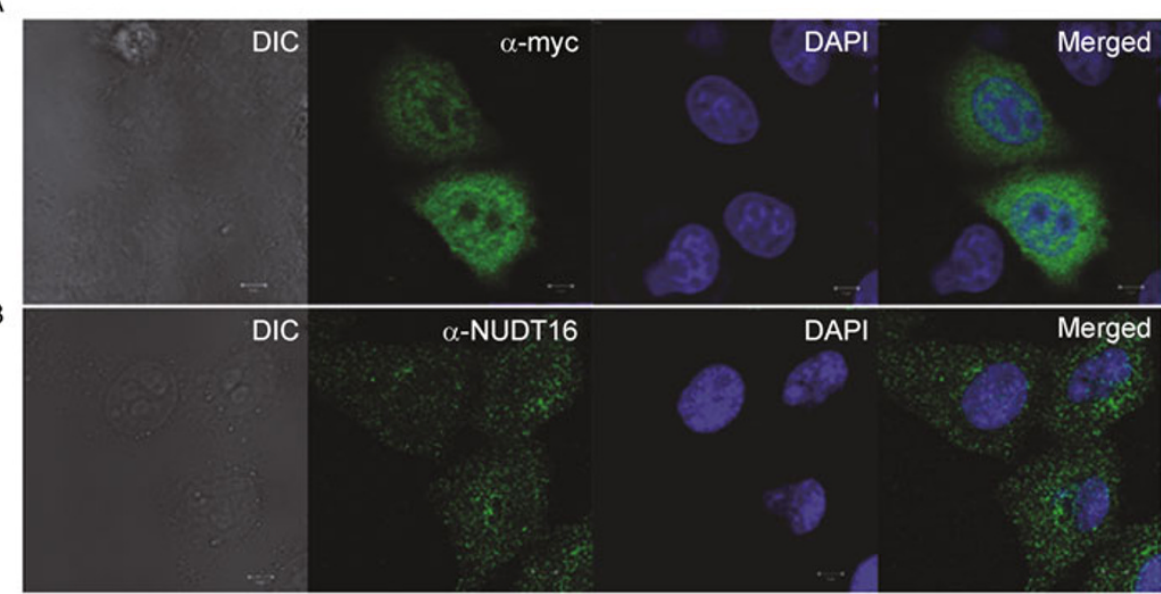

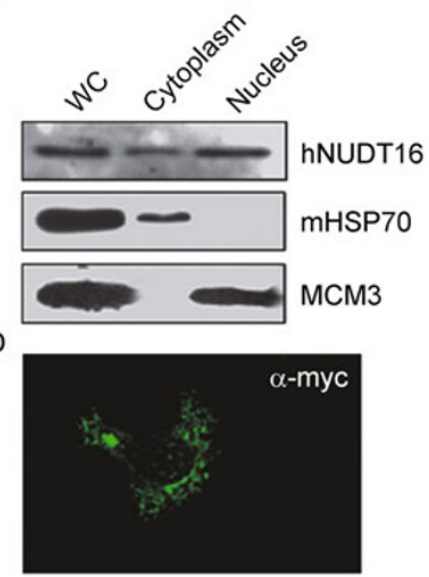

Figure 5. hNUDT16 is ubiquitously present throughout cell. (A) hNUDT16 with a C-terminal myc-epitope was overexpressed in HeLa cells and detected using anti-myc antibody by confocal microscopy. The cells were permeabilized using $0.5 \%$ Triton X-100 and the nucleus was stained with DAPI. The presented images are representative of $>90 \%$ of the transfected cells. (B) Detection of the endogenous hNUDT16 in HeLa cells with polyclonal NUDT16 anti-serum. (C) The cytoplasm and nuclear fractionations from 293T cells were prepared and loaded onto SDS-PAGE with equal amounts of the total input proteins. The relative amounts of hNUDT16 protein in the cyto- and the nucle-fractions were determined by Western-blot analysis. MCM3 and mitochondrial HSP70 were used as nuclear and cytoplasmic markers, respectively. (D) Detection of the myc-tagged hNUDT16 as in (A) except that the cells were treated with $0.1 \%$ Triton X-100.

substitution of another E92 by $Q$ also dramatically decreased the enzyme's decapping efficiency by about 6 fold (Peculis et al., 2007). As expected, similar results were also obtained for our protein (see RESULTS).

As for other Nudix hydrolases, besides these glutamic acid residues, both structural and bio-chemical studies show that the arginine residue within the motif is also actively involved in the coordination of the divalent cations, and thereby affects catalysis. In the subfamily of Mut pyrophosphahydrolase, whose catalysis mechanism has been elaborately elucidated, a significant contribution of this arginine to catalysis has been demonstrated to be around $10^{2}-10^{3}$ fold (Mildvan et al., 
2005). Whereas, the function of this conserved arginine for members of the NUDT16/X29 protein subfamily remains unclear. In this study, we confirmed that this basic residue was also vital to efficient hydrolysis. After substitution of the residue by leucine, only tiny amounts of $m^{7} G D P$ molecule could be cleaved in the standard decapping assay (see RESULTS). In light of the conservation of this arginine for NUDT16/X29 proteins, we believe that the indispensability of the amino acid in catalysis could also be extrapolated to other U8 snoRNA decapping enzymes.

\section{Stringent product specificity but loose substrate specificity for hNUDT16}

It should be noted that X29 also exhibits mRNA decapping activity in the presence of $\mathrm{Mg}^{2+}$ (Ghosh et al., 2004). Nevertheless, hNUDT16 shows highly stringent product specificities for mRNA substrates compared to its Xenopus counterpart, since, as with U8, $\mathrm{m}^{7} \mathrm{GDP}$ is the only cleaved product detected in our assay (see RESULTS; Fig. 1 and 2). However for X29, it has been demonstrated that GMP, as well as $\mathrm{m}^{7} \mathrm{GDP}$, is the decapped products when NO38 mRNA is incubated with the enzyme (Ghosh et al., 2004). Despite the high sequence similarities between the two enzymes (over $50 \%$ in identity), the difference in product specificity observed here in this study might allude to the possibility that during evolution, the human homolog evolved to behave with stringent product specificity but loose substrate specificity in order to meet the requirement of fine modulations in Homo sapiens. In support of this, a recent report by Nona Abolhassani et al. has demonstrated that hNUDT16 could also recognize and hydrolyze dIDP/IDP and therefore plays an important protective role in eliminating aberrant dIDP/IDP and dITP/ITP from nucleotide pools in mammals (Abolhassani et al., 2010). These findings reveal the versatile functions of this RNA decapping enzyme.

\section{The demonstration of mRNA decapping activity for hNUDT16 reveals its pleiotropic activity}

During preparation of this manuscript, Song et al. reported similar result about the mRNA decapping activity of hNUDT16 (Song et al., 2010). They claimed that the protein is mainly cytoplasm-localized. Nevertheless, in the present study, we found that the enzyme is of ubiquitous presence throughout the cell. We believe this distribution pattern is more reasonable since there are functions of this enzyme that require the protein to be present in nucleus (lyama et al., 2010). By chance, we found that the concentration of Triton X-100, which we used to permeabilize cells, could dramatically influence the immunofluorescent results. The abundant presence of hNUDT16 in nucleus could only be observed when the cells were treated with a high concentration $(0.5 \%)$ of Triton X-100. We believe that this phenomenon is due to the fact that the nuclear membrane is not as easily accessible to the permeabilizing reagent as the cell membrane is. Therefore a high concentration of Triton X-100 is required to fully permeabilize the nuclear membrane, thereby allowing the free entry of different antibodies into the nucleus. The discrepancy observed in this study and in the report by Song et al., in terms of the subcellular localization of hNUDT16 enzyme, may lie in the difference in the type and/or the concentration of the permeabilizing reagent used for cell treatment.

In summary, previously reported snoRNA-decapping activity of hNUDT16, together with experimental evidences proving the presence of the enzyme in nucleus, reveals the important roles the protein should play in the process of ribosome maturation (Tomasevic and Peculis, 1999; Ghosh et al., 2004); in this study, the identification of mRNA-decapping activity of this enzyme and the demonstration of its ubiquitous distribution indicate that hNUDT16 should function more actively than we previously expected by directly modulating the stability and half-life of mRNAs.

In vivo, there must be a highly elaborate machinery to maintain the homeostasis of RNA. The RNA decay and turnover system is without doubt an organic and indispensable part of this RNA metabolic chain. In this process, hNUDT16 should play an important role by acting as a universal decapping enzyme for both small nucleolar RNAs and cytoplasmic mRNAs.

\section{MATERIALS AND METHODS}

\section{Expression and purification of wild-type hNUDT16}

The DNA fragment encoding full-length hNUDT16 was obtained by PCR amplification using human kidney cDNA as a template and the following primer pairs: 5'-GGAATTCCATATGATGGCTGGTG CTCGTCGTCTGGAGCTAGGCGAG-3'; 5'-ATAGTTTAGCGGC CGCCTAGTGGTGGTGGTGGTGATGAGCTGGAATC-3'. The resultant PCR product was cloned into the Ndel and Notl restriction sites of the $\mathrm{pET}-30 \mathrm{a}$ vector. The $\mathrm{C}$-terminal His tag was introduced into the recombinant hNUDT16 by primer.

For protein expression, $1 \mu \mathrm{L}$ of the plasmid was transformed into $E$. coli BL-21(DE3) competent cells from which cultures were grown to an $\mathrm{OD}_{600}=0.8$ in LB medium supplemented with kanamycin and induced with $0.1 \mathrm{mM} \mathrm{IPTG}$ for $5 \mathrm{~h}$ at $37^{\circ} \mathrm{C}$. The cells were harvested and resuspended in cold lysis buffer (20 mM Tris- $\mathrm{HCl} \mathrm{pH} \mathrm{8.0,150} \mathrm{mM}$ $\mathrm{NaCl}$, and $5 \%$ glycerol) and homogenized by sonication. Cell debris was removed by centrifugation at 16,000 rotation/min for $30 \mathrm{~min}$. The resultant supernatant was added to Ni-NTA resin (GE) and gently mixed at $4^{\circ} \mathrm{C}$ for $1 \mathrm{~h}$. After removal of the non-specific contaminants using 20 column volume of lysis buffer, the recombinant hNUDT16 was eluted with $150 \mathrm{mM}$ imidazole in lysis buffer and concentrated to about $1 \mathrm{~mL}$. This eluant was further purified by gel filtration using Superdex 75 Hiload 16/60 (GE). The fractions containing hNUDT16 were pooled and concentrated to approximately $1 \mathrm{mg} / \mathrm{mL}$. 


\section{Site-directed mutagenesis}

The plasmid encoding wild-type hNUDT16 was used as template to generate the constructs coding for mutant enzymes dubbed R75L, $E 76 Q, E 79 Q$ and $E 80 Q$ respectively. With the following primer pairs, all the four mutant constructs were successfully obtained using the Phusion Site-mutagenesis Kit (NEB) according to the manufacturer's instructions.

R75Lf: AGGACGGGCTGAACCTCGAGCTGCGCGAGGA; R75Lr: TCCTCGCGCAGCTCGAGGTTCAGCCCGTCCT; E76Qf: GACGGGCTGAACCGCCAGCTGCGCGAGGAGC; E76Qr: GCTCCTCGCGCAGCTGGCGGTTCAGCCCGTC; E79Qf: AACCGCGAGCTGCGCCAGGAGCTGGGCGAAG; E79Qr: CTTCGCCCAGCTCCTGGCGCAGCTCGCGGTT; E80Qf: CGCGAGCTGCGCGAGCAGCTGGGCGAAGCGG; E80Qr: CCGCTTCGCCCAGCTGCTCGCGCAGCTCGCG;

The resultant sequencing-verified plasmids were transformed into BL-21 cells and the detailed protocols for the expression and purification of the mutant enzymes followed that for the wild-type protein.

\section{in vitro mRNA transcription}

A primer containing the T7 promoter was designed and used to amplify the U8 DNA using pSP6-U8 as template (the pSP6-U8 vector containing the U8 cDNA was kindly provided by Dr. Kazimierz Tycowski). The luciferase gene was supplied as the positive control in the Promega in vitro transcription kit. The gene of H5N1 influenza NP protein was used as template to amplify DNA fragment containing T7 promoter sequence (incorporated by primer) at the 5 '-end. The transcripts of unlabelled and uncapped U8, luciferase and influenza NP RNAs were obtained by in vitro transcription using T7 RNA polymerase following the manufacturer's instructions. RNAs were purified and cap-labeled with $5^{\prime} \mathrm{m}^{7} \mathrm{G}$ using the vaccinia virus capping enzyme (guanylyl tranferase, Ambion), SAM and $\left[\alpha-{ }^{32} \mathrm{P}\right] \mathrm{GTP}$ (3000 mmole/Ci, Amersham Pharmacia Biotech) as described previously (Tomasevic and Peculis, 1999).

\section{in vitro decapping assay}

For the standard decapping assay, the $\mathrm{m}^{7} \mathrm{GDP}$ was cleaved from RNAs (U8 or luciferase mRNA or mRNA encoding NP protein of influenza) by $0.3 \mu \mathrm{g}$ of either wild-type hNUDT16 or any one of the four mutant enzymes (dissolved in $20 \mathrm{mM}$ Tris- $\mathrm{HCl} \mathrm{pH} \mathrm{8.0,50} \mathrm{mM}$ $\mathrm{NaCl}$ to a final concentration of $0.3 \mathrm{mg} / \mathrm{mL}$ ) at $37^{\circ} \mathrm{C}$ for $30 \mathrm{~min}$ in a final volume of $20 \mu \mathrm{L}$ buffer containing $50 \mathrm{mM}$ Tris ( $\mathrm{pH} 8.5$ ), $150 \mathrm{mM} \mathrm{NaCl}$, $5 \%$ glycerol, and $2 \mathrm{mM} \mathrm{MgCl}_{2}$. The reactions were stopped by addition of $50 \mathrm{mM}$ EDTA and $4 \mu \mathrm{L}$ sample was blotted onto PEIcellulose (Merck \& Co. Inc., USA). The resultant TLC plates were developed in $0.75 \mathrm{M} \mathrm{LiCl}$ and $1 \mathrm{M}$ formic acid, and then dried and exposed to X-ray photographic plates (Kodak) overnight.

The modified standard decapping assay followed the protocol of the standard assay except that the $\mathrm{MgCl}_{2}$ within the reaction buffer was eliminated or replaced by $0.5 \mathrm{mM} \mathrm{MnCl}$ or $\mathrm{CoCl}_{2}$ or $\mathrm{ZnCl}_{2}$.

For the quantitative decapping assay, the amount of the input RNA (U8 or luciferase mRNA) was calculated and set at $0.2 \mathrm{nmol}$ exactly. The reaction buffer was the same as in the standard assay, but the amount of the enzyme added was raised to $0.6 \mu \mathrm{g}$. At three different time points $(15,30$, and $60 \mathrm{~min}), 5 \mu \mathrm{L}$ of the reaction solution was pooled and the reaction stopped immediately for both substrates. The resultant samples were blotted, developed and autoradiographically operated as in the standard assay.

\section{Cross-inhibition assay of RNA decapping by hNUDT16}

A standard decapping reaction was set up, except that prior to the addition of the protein, the uncapped luciferase mRNA or uncapped U8 snoRNA was added to the reaction and mixed gently. The final inhibitor/substrate molar ratio was set at 20 -fold and 2 -fold respectively. After incubation at $37^{\circ} \mathrm{C}$ for $30 \mathrm{~min}, 4 \mu \mathrm{L}$ of reaction sample was blotted on TLC plate and developed as described above. The exposure of the X-ray photographic plates was performed overnight.

\section{Generation of hNUDT16 polyclonal anti-serum}

hNUDT16 specific anti-serum was generated by immunizing BALB/c mice with His-tagged wild-type protein at the Animal Service Facility, Institute of Genetics and Developmental Biology, Chinese Academy of Sciences.

\section{Immunofluorescence}

The hNUDT16 gene was subcloned into pcDNA4/myc-His vector via BamH1/Not1 restriction sites with the following primer pairs, NUDT-F: CGGGATCCCGATGGCCGGAGCCCGCAGG; NUDT-R: ATTTG CGGCCGCTTGTGATGAGCTGGAATCT.

This recombinant plasmid will yield hNUDT16 proteins containing C-terminal myc-epitope in transfected cells.

The transient transfection of HeLa cells was conducted with the lipofectamine 2000 transfection reagent (Invitrogen) following the manufacturer's instructions. Immunofluorescence was used to detect the distribution of hNUDT16 in cells according to a standard procedure. Briefly, After $24 \mathrm{~h}$ of transfection, cells were fixed for $15 \mathrm{~min}$ with $3 \%$ paraformaldehyde. The cells were permeabilized by incubation with $0.1 \%$ or $0.5 \%$ Triton X-100 in PBS for $5 \mathrm{~min}$, and then incubated with anti-myc monoclonal antibody (Santa Cruz) or antihNUDT16 polyclonal antibody for $1 \mathrm{~h}$. The fixed cells were then washed in PBS and incubated with Alexa 488 fluor-conjugated secondary antibody (Invitrogen). Nucleus was stained by DAPI for $5 \mathrm{~min}$. The images were obtained with laser confocal microscopy (Zeiss LSM 710).

\section{Fractionation of nuclear and cytoplasm proteins and Western-blot assay}

Western blotting was used to estimate the level of endogenous hNUDT16 expression in nucleus and cytoplasm of 293T cells. Extraction of nucleus and cytoplasm was conducted with NE-PER ${ }^{\circledR}$ Nuclear and Cytoplasmic Extraction Reagents (Pierce) following the manufacturer's instructions. Western blot assay was carried out as previously reported (Mi et al., 2010). Samples containing $10 \mu \mathrm{g}$ proteins were loaded on SDS-PAGE, and then transferred to nitrocellulose membranes. The hNUDT16 was identified with antihNUDT16 polyclonal antibody probe using an enhanced chemiluminescence system (Pierce). 


\section{ACKNOWLEDGEMENTS}

The pSP6-U8 vector containing the U8 cDNA was a generous gift from Dr. Kazimierz Tycowski (Howard Hughes Medical Institute, Yale University School of Medicine). We thank Mr. Joel Haywood and Dr. Christopher J. Vavricka for their help and comments on the manuscript. This work was supported by the Natural Science Foundation of China (No. 30870118)

\section{REFERENCES}

Abolhassani, N., Iyama, T., Tsuchimoto, D., Sakumi, K., Ohno, M., Behmanesh, M., and Nakabeppu, Y. (2010). NUDT16 and ITPA play a dual protective role in maintaining chromosome stability and cell growth by eliminating dIDP/IDP and dITP/ITP from nucleotide pools in mammals. Nucleic Acids Res 38, 2891-2903.

Anderson, J.S., and Parker, R.P. (1998). The 3' to 5' degradation of yeast mRNAs is a general mechanism for mRNA turnover that requires the SKI2 DEVH box protein and $3^{\prime}$ to $5^{\prime}$ exonucleases of the exosome complex. EMBO J 17, 1497-1506.

Bessman, M.J., Frick, D.N., and O'Handley, S.F. (1996). The MutT proteins or "Nudix" hydrolases, a family of versatile, widely distributed, "housecleaning" enzymes. J Biol Chem 271, 25059-25062.

Bhatnagar, S.K., Bullions, L.C., and Bessman, M.J. (1991). Characterization of the mutT nucleoside triphosphatase of Escherichia coli. J Biol Chem 266, 9050-9054.

Chen, C.Y., Gherzi, R., Ong, S.E., Chan, E.L., Raijmakers, R., Pruijn, G.J., Stoecklin, G., Moroni, C., Mann, M., and Karin, M. (2001). AU binding proteins recruit the exosome to degrade ARE-containing mRNAs. Cell 107, 451-464.

Coller, J., and Parker, R. (2004). Eukaryotic mRNA decapping. Annu Rev Biochem 73, 861-890.

Cougot, N., Babajko, S., and Séraphin, B. (2004a). Cytoplasmic foci are sites of mRNA decay in human cells. J Cell Biol 165, 31-40.

Cougot, N., van Dijk, E., Babajko, S., and Séraphin, B. (2004b). 'Captabolism'. Trends Biochem Sci 29, 436-444.

Das, B., Butler, J.S., and Sherman, F. (2003). Degradation of normal mRNA in the nucleus of Saccharomyces cerevisiae. Mol Cell Biol 23, 5502-5515.

Das, B., Das, S., and Sherman, F. (2006). Mutant LYS2 mRNAs retained and degraded in the nucleus of Saccharomyces cerevisiae. Proc Natl Acad Sci U S A 103, 10871-10876.

Das, B., Guo, Z., Russo, P., Chartrand, P., and Sherman, F. (2000). The role of nuclear cap binding protein Cbc1p of yeast in mRNA termination and degradation. Mol Cell Biol 20, 2827-2838.

Decker, C.J., and Parker, R. (1994). Mechanisms of mRNA degradation in eukaryotes. Trends Biochem Sci 19, 336-340.

Dostie, J., Lejbkowicz, F., and Sonenberg, N. (2000). Nuclear eukaryotic initiation factor $4 \mathrm{E}$ (elF4E) colocalizes with splicing factors in speckles. J Cell Biol 148, 239-247.

Dunckley, T., and Parker, R. (1999). The DCP2 protein is required for mRNA decapping in Saccharomyces cerevisiae and contains a functional MutT motif. EMBO J 18, 5411-5422.

Dunckley, T., and Parker, R. (2001). Yeast mRNA decapping enzyme. Methods Enzymol 342, 226-233.

Fenger-Grøn, M., Fillman, C., Norrild, B., and Lykke-Andersen, J. (2005). Multiple processing body factors and the ARE binding protein TTP activate mRNA decapping. Mol Cell 20, 905-915.

Fisher, D.I., Cartwright, J.L., Harashima, H., Kamiya, H., and McLennan, A.G. (2004). Characterization of a nudix hydrolase from Deinococcus radiodurans with a marked specificity for (deoxy)ribonucleoside 5'-diphosphates. BMC Biochem 5, 7.

Garneau, N.L., Wilusz, J., and Wilusz, C.J. (2007). The highways and byways of mRNA decay. Nat Rev Mol Cell Biol 8, 113-126.

Ghosh, T., Peterson, B., Tomasevic, N., and Peculis, B.A. (2004). Xenopus U8 snoRNA binding protein is a conserved nuclear decapping enzyme. Mol Cell 13, 817-828.

Gu, M., Fabrega, C., Liu, S.W., Liu, H., Kiledjian, M., and Lima, C.D. (2004). Insights into the structure, mechanism, and regulation of scavenger mRNA decapping activity. Mol Cell 14, 67-80.

Hori, M., Fujikawa, K., Kasai, H., Harashima, H., and Kamiya, H. (2005). Dual hydrolysis of diphosphate and triphosphate derivatives of oxidized deoxyadenosine by Orf17 (NtpA), a MutT-type enzyme. DNA Repair (Amst) 4, 33-39.

Ito, R., Hayakawa, H., Sekiguchi, M., and Ishibashi, T. (2005). Multiple enzyme activities of Escherichia coli MutT protein for sanitization of DNA and RNA precursor pools. Biochemistry 44, 6670-6674.

lyama, T., Abolhassani, N., Tsuchimoto, D., Nonaka, M., and Nakabeppu, Y. (2010). NUDT16 is a (deoxy)inosine diphosphatase, and its deficiency induces accumulation of single-strand breaks in nuclear DNA and growth arrest. Nucleic Acids Res 38, 4834-4843.

Kuai, L., Das, B., and Sherman, F. (2005). A nuclear degradation pathway controls the abundance of normal mRNAs in Saccharomyces cerevisiae. Proc Natl Acad Sci U S A 102, 13962-13967.

Lykke-Andersen, J. (2002). Identification of a human decapping complex associated with hUpf proteins in nonsense-mediated decay. Mol Cell Biol 22, 8114-8121.

Meyer, S., Temme, C., and Wahle, E. (2004). Messenger RNA turnover in eukaryotes: pathways and enzymes. Crit Rev Biochem Mol Biol 39, 197-216.

Mi, S., Li, Y., Yan, J., and Gao, G.F. (2010). Na(+)/K (+)-ATPase $\beta 1$ subunit interacts with $M 2$ proteins of influenza $A$ and $B$ viruses and affects the virus replication. Sci China Life Sci 53, 1098-1105.

Mildvan, A.S., Xia, Z., Azurmendi, H.F., Saraswat, V., Legler, P.M., Massiah, M.A., Gabelli, S.B., Bianchet, M.A., Kang, L.W., and Amzel, L.M. (2005). Structures and mechanisms of Nudix hydrolases. Arch Biochem Biophys 433, 129-143.

Mukherjee, D., Gao, M., O'Connor, J.P., Raijmakers, R., Pruijn, G., Lutz, C.S., and Wilusz, J. (2002). The mammalian exosome mediates the efficient degradation of mRNAs that contain AU-rich elements. EMBO J 21, 165-174.

Parker, R., and Song, H. (2004). The enzymes and control of eukaryotic mRNA turnover. Nat Struct Mol Biol 11, 121-127.

Peculis, B.A., Reynolds, K., and Cleland, M. (2007). Metal determines efficiency and substrate specificity of the nuclear NUDIX decapping proteins X29 and H29K (Nudt16). J Biol Chem 282, 24792-24805.

Peculis, B.A., and Steitz, J.A. (1993). Disruption of U8 nucleolar snRNA inhibits $5.8 \mathrm{~S}$ and $28 \mathrm{~S}$ rRNA processing in the Xenopus oocyte. Cell 73, 1233-1245.

Piccirillo, C., Khanna, R., and Kiledjian, M. (2003). Functional characterization of the mammalian mRNA decapping enzyme hDcp2. RNA 9, 1138-1147.

Saguez, C., Olesen, J.R., and Jensen, T.H. (2005). Formation of 
export-competent mRNP: escaping nuclear destruction. Curr Opin Cell Biol 17, 287-293.

Scarsdale, J.N., Peculis, B.A., and Wright, H.T. (2006). Crystal structures of U8 snoRNA decapping nudix hydrolase, X29, and its metal and cap complexes. Structure 14, 331-343.

Sharma, S., and Black, D.L. (2006). Maps, codes, and sequence elements: can we predict the protein output from an alternatively spliced locus? Neuron 52, 574-576.

Song, M.G., Li, Y., and Kiledjian, M. (2010). Multiple mRNA decapping enzymes in mammalian cells. Mol Cell 40, 423-432.

Taylor, M.J., and Peculis, B.A. (2008). Evolutionary conservation supports ancient origin for Nudt16, a nuclear-localized, RNAbinding, RNA-decapping enzyme. Nucleic Acids Res 36, 6021-6034.

Tomasevic, N., and Peculis, B. (1999). Identification of a U8 snoRNAspecific binding protein. J Biol Chem 274, 35914-35920.

Tucker, M., and Parker, R. (2000). Mechanisms and control of mRNA decapping in Saccharomyces cerevisiae. Annu Rev Biochem 69,
571-595.

van Dijk, E., Cougot, N., Meyer, S., Babajko, S., Wahle, E., and Séraphin, B. (2002). Human Dcp2: a catalytically active mRNA decapping enzyme located in specific cytoplasmic structures. EMBO J 21, 6915-6924.

Vasudevan, S., and Peltz, S.W. (2003). Nuclear mRNA surveillance. Curr Opin Cell Biol 15, 332-337.

Wang, Z., Jiao, X., Carr-Schmid, A., and Kiledjian, M. (2002). The $\mathrm{hDcp} 2$ protein is a mammalian mRNA decapping enzyme. Proc Natl Acad Sci U S A 99, 12663-12668.

Wang, Z., and Kiledjian, M. (2001). Functional link between the mammalian exosome and mRNA decapping. Cell 107, 751-762.

Wilusz, C.J., and Wilusz, J. (2004). Bringing the role of mRNA decay in the control of gene expression into focus. Trends Genet 20, $491-497$.

Zhang, J., Gao, F., Zhang, Q., Chen, Q., Qi, J., and Yan, J. (2008). Crystallization and crystallographic analysis of human NUDT16. Acta Crystallogr Sect F Struct Biol Cryst Commun 64, 639-640. 